\title{
A versatile microfluidic device for multiple ex vivo/ in vitro tissue assays unrestrained from tissue topography
}

\author{
Jose M. de Hoyos-Vega (1)', Alan M. Gonzalez-Suarez (10 ${ }^{1}$ and Jose L. Garcia-Cordero (iD ${ }^{1}$
}

\begin{abstract}
Precision-cut tissue slices are an important in vitro system to study organ function because they preserve most of the native cellular microenvironments of organs, including complex intercellular connections. However, during sample manipulation or slicing, some of the natural surface topology and structure of these tissues is lost or damaged. Here, we introduce a microfluidic platform to perform multiple assays on the surface of a tissue section, unhindered by surface topography. The device consists of a valve on one side and eight open microchannels located on the opposite side, with the tissue section sandwiched between these two structures. When the valve is actuated, eight independent microfluidic channels are formed over a tissue section. This strategy prevents cross-contamination when performing assays and enables parallelization. Using irregular tissues such as an aorta, we conducted multiple in vitro and ex vivo assays on tissue sections, including short-term culturing, a drug toxicity assay, a fluorescence immunohistochemistry staining assay, and an immune cell assay, in which we observed the interaction of neutrophils with lipopolysaccharide (LPS)-stimulated endothelium. Our microfluidic platform can be employed in other disciplines, such as tissue physiology and pathophysiology, morphogenesis, drug toxicity and efficiency, metabolism studies, and diagnostics, enabling the conduction of several assays with a single biopsy sample.
\end{abstract}

\section{Introduction}

Tissue slices are sections of tissues harvested in vivo, excised, sliced, explanted and cultured in vitro. Tissue slices were first introduced in 1923, but it was not until 1980 that interest was revived with the invention of a slicer that allowed tissues with a defined thickness and uniformity to be prepared (precision-cut tissue slices) ${ }^{1}$. Slices are commonly obtained with a vibratome, microtome or a tissue chopper ${ }^{2}$ but can also be obtained manually with a scalpel ${ }^{3}$ or a needle puncher ${ }^{4}$. Section thicknesses can range from $4 \mu \mathrm{m}$ to $2 \mathrm{~mm}^{5,6}$, although the most common thickness for ex vivo studies ranges from 100 to $400 \mu \mathrm{m}^{7,8}$. Tissue-cut slices resemble the in vivo histology of an organ because they retain organ cytoarchitecture (to a certain extent), contain the different types of cells that make up an organ, maintain organ

\footnotetext{
Correspondence: Jose L. Garcia-Cordero (jlgarciac@cinvestav.mx)

'Unidad Monterrey, Centro de Investigación y de Estudios Avanzados del IPN, Via del conocimiento 201, Parque PIIT, Apodaca, NL 66628, Mexico
}

matrix configuration ${ }^{2,3,9}$ and preserve metabolic enzymes, transporters, and cofactors ${ }^{10}$. In some cases, tissue slices have been shown to better predict in vivo metabolite profiles than cell lines and can quantitatively predict drug metabolite clearance ${ }^{3}$.

Classic techniques for organotypic slice culture include roller tube cultures, membrane cultures, and petri dishes ${ }^{2}$. Tissue-cut slices from the liver, kidney, lung, brain, glands, heart, prostate, and spleen have been used in a wide range of applications including drug metabolism and discovery, physiology, morphology, development, endocrinology, and toxicology ${ }^{2,9,10}$. Most tissue slices can be cultured for up to $24 \mathrm{~h}$; however, using dynamic organ culture incubation systems with appropriate culture media, oxygen supply conditions, and media perfusion, this period can be extended for up to 7 days $^{3}$ or even weeks to months ${ }^{2}$. Slices of different organs are prepared using similar straightforward techniques ${ }^{10}$. However, a major limitation of these techniques is that they require a 


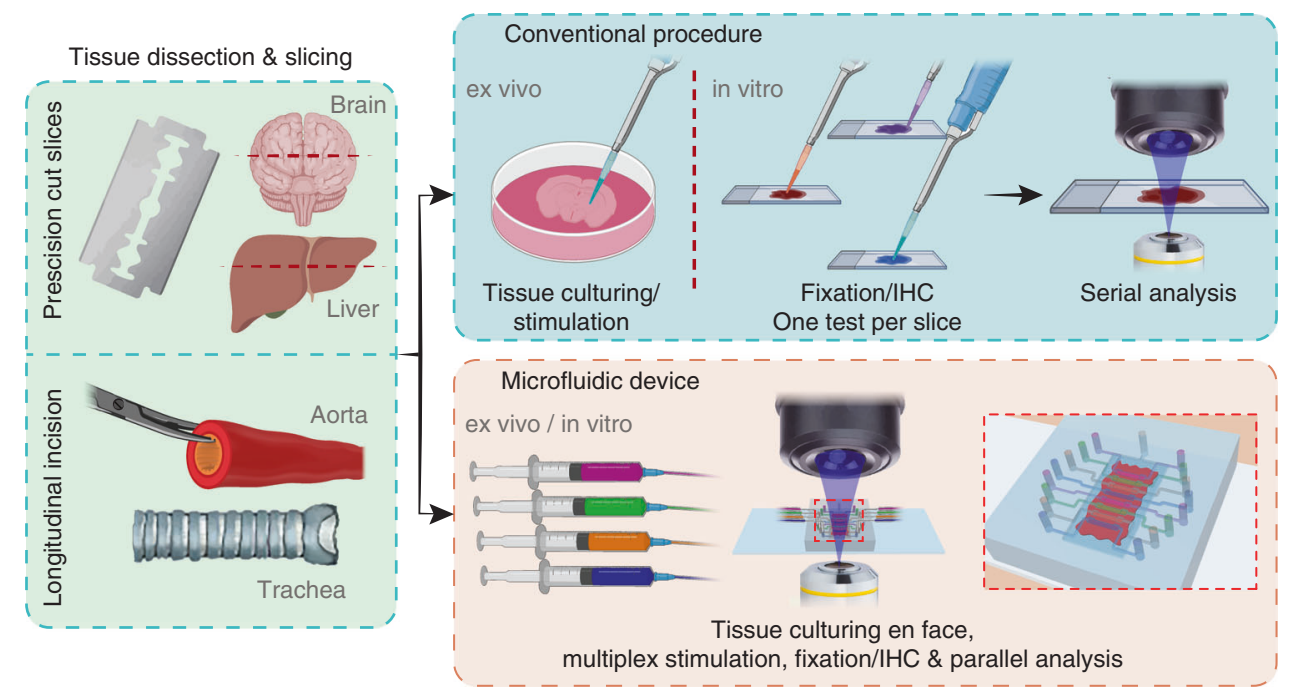

Fig. 1 Comparison of the conventional procedure and our microfluidic device to analyze tissue slices. Top panel: Dissected tissue samples are sliced and cultivated in a static environment with a single stimulation. Samples are later fixed and stained on a glass slide for downstream serial analysis. Bottom panel: Tissue samples are placed in the microfluidic device, allowing for a dynamic culturing environment, multiple parallel stimulation, immunohistochemical $(\mathrm{HC})$ staining, and analysis

single tissue sample for each biochemical assay, increasing the demand for samples that may be scarce (e.g., biopsies), notwithstanding the large volumes of media and reagents used $^{11-15}$ (Fig. 1). Therefore, new technologies that perform multiple assays with a single tissue section will have tremendous impact on the fields of pharmacology, toxicology, tissue physiology, and pathophysiology, and diagnostics 9 . Ultimately, tissue slices can become an important tool to test drug efficacy, safety, and toxicology in humans before clinical trials.

Tissues are surrounded by connective tissue that has a key role in providing structure, repair, homeostasis, extracellular matrix production, nutrient supply, cell signaling and cell proliferation ${ }^{16,17}$. The removal of connective tissue alters ex vivo cellular responses. However, the connective tissue has a highly irregular composition: a variable thickness, an uneven surface, and different stiffness, cellular composition, and shape. These features hinder tissue analysis and are commonly removed to provide a smooth, flat tissue surface ${ }^{7,13}$. Furthermore, slicing tissues with a vibratome damages the outer layers of the tissue due to mechanical stress, requiring at least $1 \mathrm{~h}$ of incubation before an assay can be performed ${ }^{18}$. Thus, it is important to preserve the connective tissue to obtain a physiological response closer to in vivo.

Culturing tissue sections with irregular surfaces has been attempted in microfluidic devices, however, the tissue irregularities quickly lead to high reagent diffusion and cross-contamination ${ }^{13,18-21}$. Other microfluidic devices (Sup Table 1) have been reported to analyze tissue sections from different organs ${ }^{6,7,22,23}$, but they use either flat and homogenous tissue sections or fixed thin tissue slices of $\sim 5 \mu \mathrm{m}^{5,24,25}$, which renders them impractical to analyze tissue sections with irregular topographies. Thus, there is a need to develop technologies to study tissue sections ex vivo unrestrained by the physical and mechanical features of tissue sections, and versatile enough to perform multiple ex vivo and in vitro assays on a single tissue sample. These assays include fixing, staining, drug screening, introducing cells for immune studies, and evaluation of environmental risk factors, among others.

To address some of these issues, we developed a microfluidic platform that can accommodate tissue sections of varying topologies, thicknesses and organ sources. The device can perform multiple ex vivo and in vitro assays on a fresh tissue sample en face. To demonstrate the versatility of our platform, we carried out a drugresponse assay, a viability assay, immunohistochemistry staining, and tissue-immune cell interaction studies. In the latter study, we demonstrated the interaction of neutrophils with the endothelia of a tissue section after stimulation with lipopolysaccharide (LPS).

\section{Results and discussion}

\section{Tissue characteristics}

To demonstrate the highly irregular topography of some tissues, micrographs (Fig. 2) of two irregular tissue sections used in this study, the murine aorta and trachea, are shown. The cross-sectional thickness of a murine aorta can range from 44 to $111 \mu \mathrm{m}$ with an average of $74 \mu \mathrm{m}$, while the trachea thickness ranges from 76 to $171 \mu \mathrm{m}$ with 

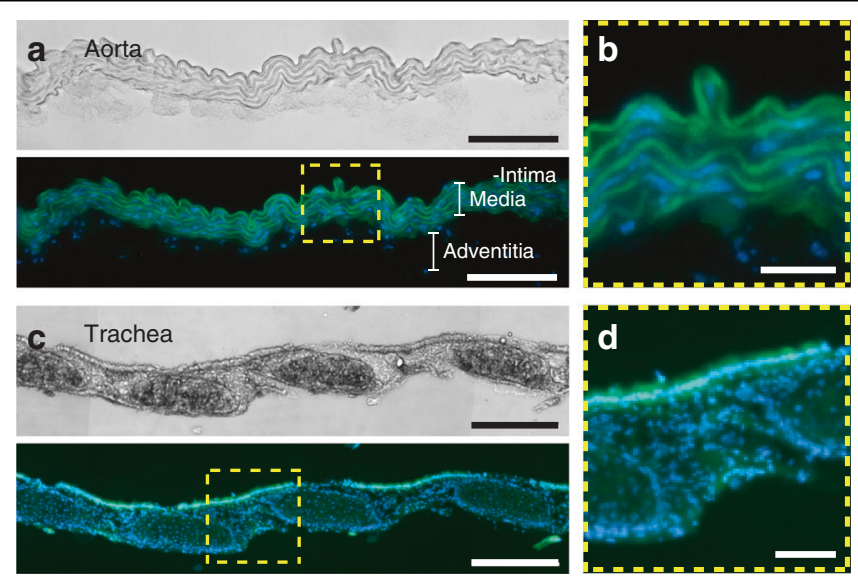

Fig. 2 Tissue characteristics of the aorta and trachea. a Brightfield and fluorescence images of a $12 \mu \mathrm{m}$ cryostat slice of the descending aorta en face showing an irregular surface and thickness profile. The tunica intima is located on the upper side of the tissue and lays on top of the tunica media composed of a thick variable layer of muscle fibers (autofluorescence). The adventitia layer can be distinguished by the scattered cells (blue, Hoechst staining) beneath the tunica media. Scale bar: left, $100 \mu \mathrm{m}$. b Magnification of the yellow square in (a). Scale bar: $25 \mu \mathrm{m}$. c Murine trachea section. Collagen rings (disk-like structures) and annular ligament segments can be identified in brightfield and fluorescence images of a murine trachea slice en face. The epithelial monolayer (green autofluorescence) is located on the top of the tissue. Scale bar: $200 \mu \mathrm{m}$. $\mathbf{d}$ Magnification of the yellow square in (c). Scale bar: $50 \mu \mathrm{m}$

an average of $127 \mu \mathrm{m}$. The aorta, with its tunica intima, media and adventitia, represents a tissue with a uniform composition but with substantial thickness variability (Fig. 2a, b). The trachea, composed of interspersed collagen rings and annular ligaments, represents an irregular tissue morphology due to its interleaved stiff and soft sections along its length (Fig. 2c, d). It is clear from these micrographs that their topography is highly irregular and very different from the flat and homogeneous sections that are commonly used in tissue section studies. Thus, the challenge for any technology is to accommodate tissue sections with variations in height and differences in stiffness.

\section{Device design and fabrication}

To work with these irregular tissue sections and perform multiple assays with an entire tissue excision, we developed a multilayer polydimethylsiloxane (PDMS) microfluidic device (Fig. 3a). The device consists of four stacked layers initially separated into two assemblies (Figs. 3b, S1). The bottom assembly comprises a valve located below an open tissue chamber, while the top assembly contains an array of eight assay channels (150- $\mu \mathrm{m}$ deep, 200- $\mu \mathrm{m}$ wide, and 1.1$\mathrm{mm}$ long) that connect to an array of top open microchannels with eight inlets and outlets. A tissue section is manually placed in the tissue chamber before sealing with the top assembly (Fig. 3c). The tissue chamber features an outlet and an inlet, through which fresh media is introduced to constantly periperfuse the tissue. Drugs and solutions are introduced through the eight channel inlets.

Based on the dimensions of the open folded thoracic descending aorta (length: $\sim 6 \mathrm{~mm}$, width: $\sim 2.5 \mathrm{~mm}$ ), the tissue chamber was designed to be $7.6 \mathrm{~mm} \times 2.9 \mathrm{~mm}$. This additional room facilitated tissue deposition. (Note that the chamber dimensions and assay microchannels can be modified according to tissue and experimental needs.) To avoid contact of the tissue and liquid with the top layer before irreversible bonding, the tissue chamber was designed to be $100 \mu \mathrm{m}$ taller than the thickest feature of the tissue (250- $\mu \mathrm{m}$ deep).

Located beneath the tissue chamber is a flexible valve with similar dimensions to the tissue chamber (Fig. 3b). When the valve is actuated, it pushes the tissue section upwards until it touches the assay microchannels, thus effectively creating enclosed channels separated from each other by $300 \mu \mathrm{m}$ (Fig. 3d, c). This arrangement enables tissue culture and experimentation under eight different conditions at the same time. Other microfluidic devices for multiplexing ex vivo studies use open chambers that contain a porous membrane where the tissue section is placed or are assembled from different materials to encapsulate the tissue in the device $^{4,12,13,19,22,24}$. In contrast to these approaches, our platform integrates all components in a monolithic PDMS device.

\section{Device characterization}

We first characterized the surface profile that the valve acquires at different pressures. In its native state, the valve is flat, but when actuated, it resembles an arch that can reach up to $630 \mu \mathrm{m}$ at its apex (Fig. S2). Because of this arch shape, the degree of tissue penetration in each of the eight channels is related to the position of the microchannel in the chamber and to the pressure applied on the valve (Fig. S3a-d). Confocal micrographs taken of the 

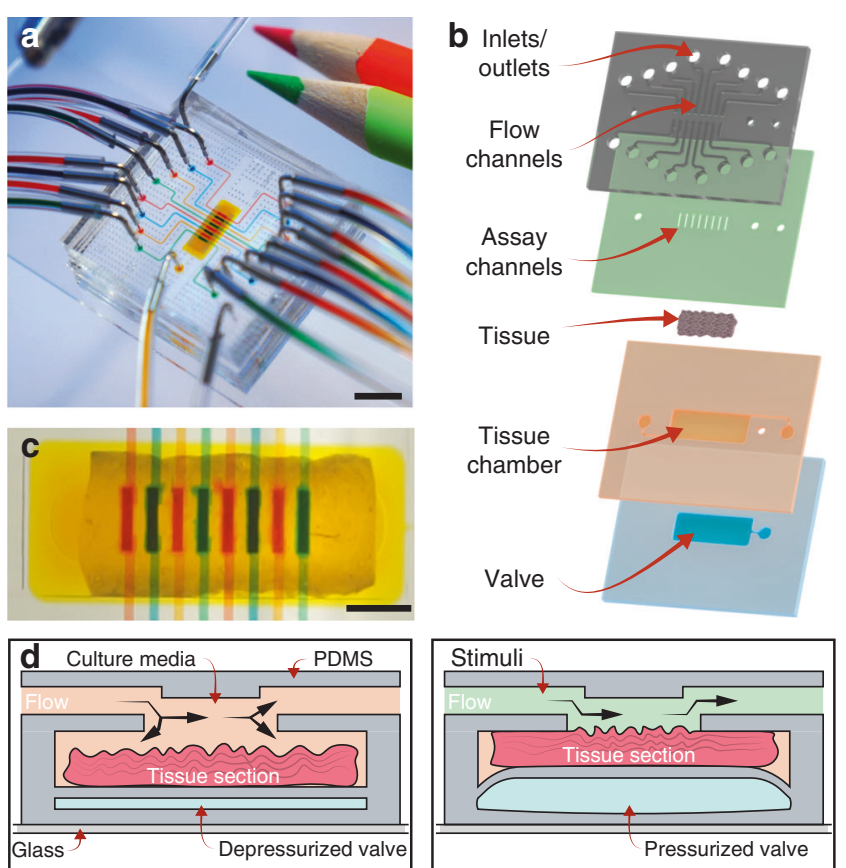

Fig. 3 Device design and working principle. a Photograph of the microfluidic platform; scale bar: $5 \mathrm{~mm}$. $\mathbf{b}$ Schematic of the assembly of the microfluidic device. The device is composed of four layers: (1) flow channels and eight inlets with their respective mirrored outlets; (2) 8 assay microchannels; (3) an open tissue chamber with an inlet and outlet; and (4) a valve. c Micrograph of a tissue section (an aorta in this case) inside the device with each assay microchannel independently perfused with different food dyes; scale bar: $1 \mathrm{~mm}$. $\mathbf{d}$ Schematic cross-sectional view of the working mechanism of the microfluidic device. The left schematic illustrates a tissue section placed on the tissue chamber with an unpressurized valve in which media flows into the tissue chamber. When the valve is actuated (right schematic), the tissue section moves up and is sealed against the assay channels, creating closed channels, where only the top surface tissue is exposed to a stimulus

middle channel, actuated at 7 and $35 \mathrm{kPa}$, show that the tissue indeed penetrates the channel in a parabolic form (Fig. S3c). It is important to consider these cross-sectional area variations along the channel, as this variation will give rise to differences in flow velocity and can affect assays that require the precise control of flow velocity (Fig. S4). In general, we found that there was good sealing between the tissue and the channels with the valve actuated at more than $7 \mathrm{kPa}$. Importantly, we did not observe any delamination even at $35 \mathrm{kPa}$.

Next, we investigated whether there was any crosstalk between the microfluidic channels or biomolecule diffusion through the tissue from one channel into neighboring channels. These experiments were carried out at $16 \mathrm{kPa}$, which is the pressure that aortic tissues experience under normal physiological conditions ${ }^{26}$. We chose three fluorescent dyes of different molecular weights representing a range of biomolecules delivered into tissues ${ }^{27,28}$ : two cell-impermeable dyes, dextran-rhodamine (40 KDa) and fluorescein isothiocyanate $(389 \mathrm{Da})$, and a cellpermeable DNA staining dye, Hoechst $(616 \mathrm{Da})$. While Hoechst flowed in all channels, the other two dyes flowed interspersed in the eight microchannels of the device. After $10 \mathrm{~min}$ of perfusion, neither dextran-rhodamine (Dextran-R) nor fluorescein isothiocyanate (FITC) leaked into adjoining microchannels, while Hoechst stained all the nuclei of the cells residing inside the channels (Fig. $4 \mathrm{a}-\mathrm{c})$. In comparison, in other microfluidic devices, leakage and intercellular diffusion of these biomolecules occurs in the first $10 \mathrm{~s}$ of perfusion, at least for brain and lymph node precision-cut slices ${ }^{13,18,21}$.

The fluorescent traces of the three dyes along the eight microchannels had similar profiles: a sharp increase in fluorescence intensity at the edges of the microchannels and a high level of fluorescence inside the width of the channel (Fig. 4d). In contrast, between the microchannels, the fluorescence levels were close to zero, indicating very little leakage. As shown in Figs. S5 and S6, only the cells inside the channels were stained. When a soft tissue is compressed, fluid in the interstitial space is expelled, preventing molecules from diffusing through these compressed regions. The solute diffusivity through a tissue depends on its physical and chemical properties and on the composition of the extracellular matrix ${ }^{29}$. These results demonstrated the ability of our device to confine the biomolecules inside the channels with no crosstalk between channels.

We also investigated the performance of our device with tissues of different stiffnesses, such as the trachea, which has softer regions. We obtained comparable fluorescence 

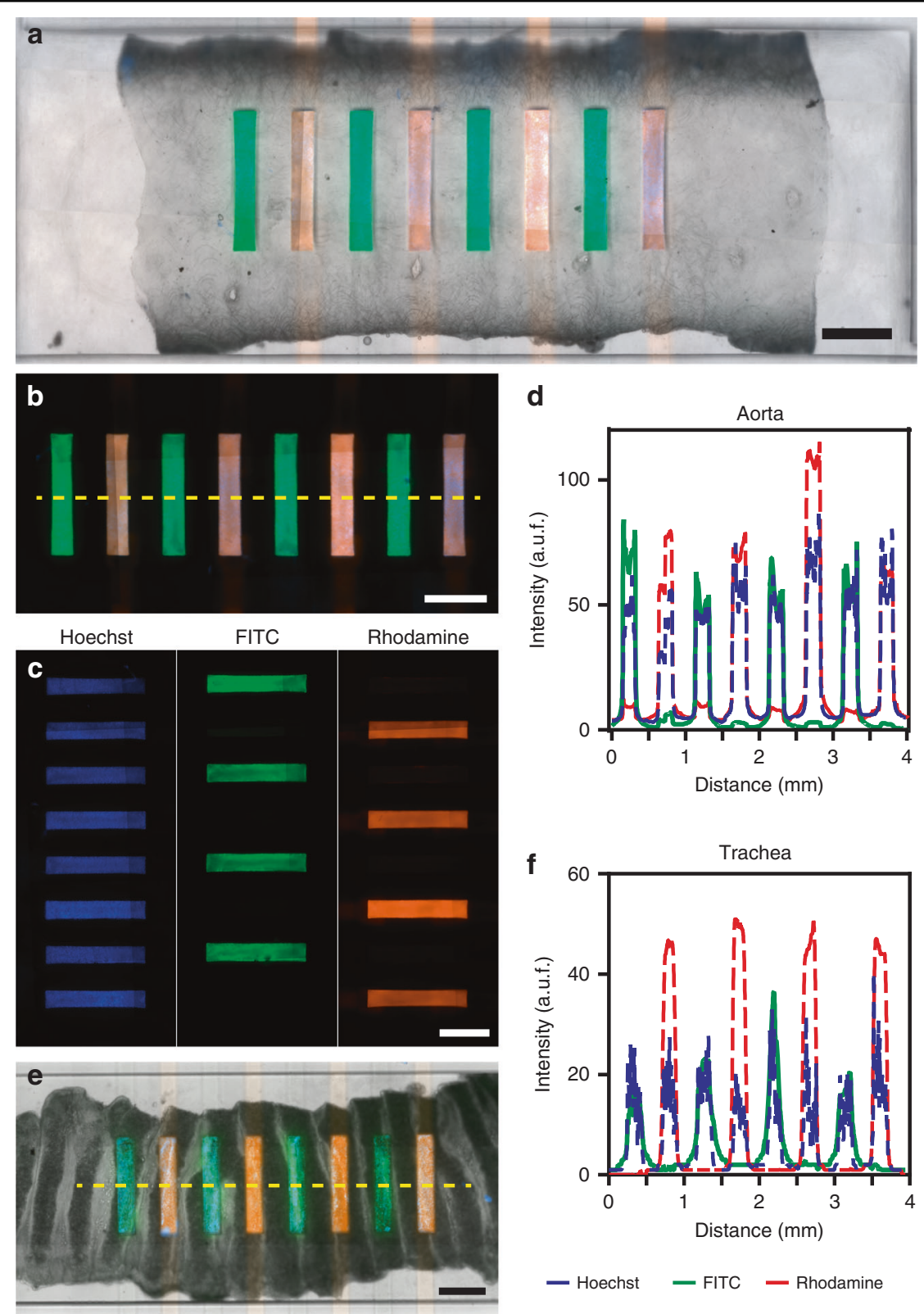

- Hoechst - FITC - Rhodamine

Fig. 4 Leakage and intercellular diffusion assay on a tissue section. a Brightfield and fluorescence micrograph of an aorta in the tissue chamber when Dextran-R or FITC was flowed in the eight microchannels in combination with Hoechst; scale bar: $1 \mathrm{~mm}$. b Fluorescence micrograph showing that fluorescence dyes are confined inside the assay microchannels; scale bar: $500 \mu \mathrm{m}$. c Micrographs of the separate fluorescence channels; scale bar: $500 \mu \mathrm{m}$. $\mathbf{d}$ Fluorescence intensity profile of the dotted line shown in image (b). e Merged brightfield and fluorescence micrograph of the trachea en face; scale bar: $500 \mu \mathrm{m}$. f Fluorescence intensity profile of the dotted line shown in image (e)

intensity profiles except for FITC, which leaked marginally in areas where the cartilage rings and annular ligaments converged at the edge of the microchannel walls (Fig. 4e, f). A possible solution to this leakage problem is to space the channels further apart and reduce the width of the microchannels to decrease the contact area of the tissue with the open microchannel. Further validation of our device should be carried out with tissue sections from other organs.

\section{Ex vivo experimentation Tissue viability}

We assessed whether a tissue section could be cultured in the microfluidic device and compared its performance to conventional static culture techniques (culture dishes) ${ }^{7}$. Tissue death was evaluated using ethidium homodimer (EthD), a membrane impermeable DNA fluorescent dye that identifies cell death and damage. As a positive control, the tissue was exposed to $0.05 \%$ Tween 20 to permeabilize 

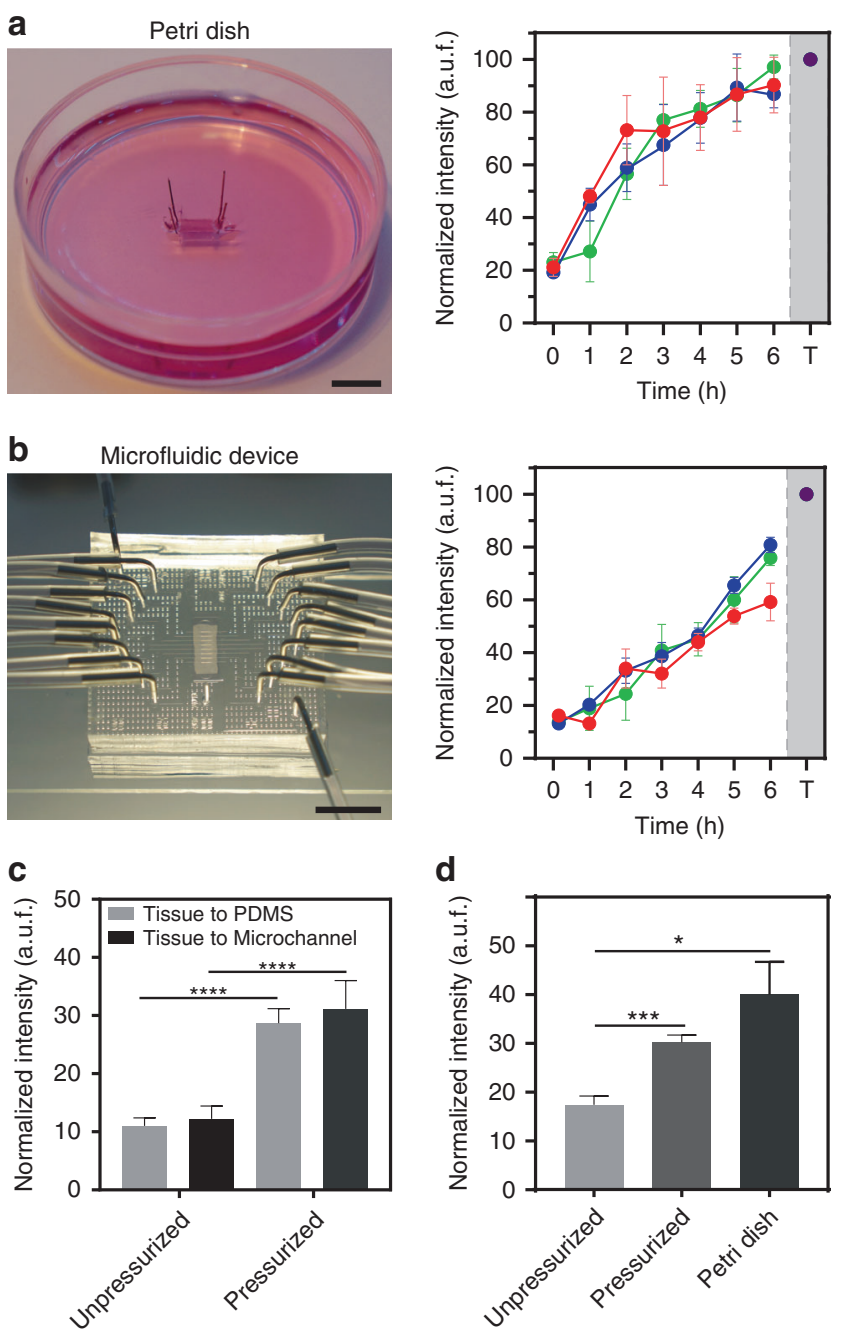

Fig. 5 Comparison of tissue viability in a Petri dish vs microfluidic device. Tissue cell death was analyzed using EthD fluorescence intensity; Tween 20 (denoted as T) was used as a positive cell death control. Graphs show the results of aortic tissue cultured for $6 \mathrm{~h}$ in (a) a conventional static petri dish and (b) in our microfluidic device with constant perfusion. Each colored line represents a different tissue section. Error bars represent at least three measurements taken in different sections of the tissue. c Comparison of tissue viability in the microchannels and the PDMS interfaces before and after pressurization in the microfluidic device. $\mathbf{d}$ Comparison of tissue viability for $1 \mathrm{~h}$ under different conditions: in a device with the valve resting (unpressurized), with the valve actuated (pressurized) and in a Petri dish. ${ }^{*}$ Indicates a $p$-value $<0.05$, and ${ }^{* * *}$ indicates a $p$-value $<0.0001$. Scale bar: $5 \mathrm{~mm}$

the cell membrane and induce cell death. An aorta section was anchored with needles on a petri dish covered with a thick layer of PDMS (Fig. 5a). The needles restricted tissue movement during media exchange and helped extend the tissue section $1 \mathrm{~mm}$ above the PDMS surface, so it was perfused with media on both sides. At the same time, we cultivated a tissue section (an aorta) in the device with continuous periperfusion for $7 \mathrm{~h}\left(37^{\circ} \mathrm{C}\right.$ with $\left.5 \% \mathrm{CO}_{2}\right)$ without valve pressurization (Fig. $5 \mathrm{~b}$ ). The cell damage caused by tissue dissection and manipulation was assessed at the beginning of each experiment $(t=0)$. Remarkably, in our microfluidic device, tissue sections showed low levels of cell death signal for the first hour compared with tissue sections in the Petri dish, which showed a sharp increase in the cell death signal. Constant perfusion of the tissue with nutrients and washing away waste products promotes cell viability, while oxygen diffusion through the gas-permeable PDMS keeps the media oxygenated ${ }^{7}$. However, after the first hour, areas of the tissue sections rapidly started to die. Long-term cultures (several days), as seen in cannulated tissues ${ }^{15}$, could not be achieved with our device or with the Petri dish. Although the constant perfusion of nutrients can diffuse through a $200-\mu \mathrm{m}$-thick tissue section and keep the tissue section alive ${ }^{7}$, the thin PDMS layer located below the tissue inhibits media perfusion to the bottom surface. It is possible to replace this 
layer with a porous membrane to deliver nutrients and increase lifespan, as already noted in other reports ${ }^{13,19}$.

Next, we performed a viability assay with the valve actuated at $16 \mathrm{kPa}$ since this is the pressure that aorta tissues experience under normal physiological conditions $^{26}$, although the valve can be actuated at higher pressures to evaluate the effects of mechanical compression on ex vivo tissues that can lead to pathological effects. With an aorta inside the device, the valve was actuated at $\sim 16 \mathrm{kPa}$ for $1 \mathrm{~h}$ while media was continuously perfused. Then, the valve was deactivated, and EthD was flowed for $10 \mathrm{~min}$ before image acquisition (Fig. S7). We observed a twofold increase in the signal intensity of cell death under $\sim 16 \mathrm{kPa}$ compared with the control conditions (before valve pressurization, Fig. 5c). This significant increase in cell death was not observed in unpressurized tissue after $1 \mathrm{~h}$ (Fig. 5b). Remarkably, the areas outside the microchannels - where the tissue is in direct contact with PDMS - presented similar levels of tissue damage as areas of tissue that were constantly perfused through the microchannels. This effect could be attributed to cell-cell junctions or to transmural interstitial flow ${ }^{30}$. Furthermore, we found that compared with no compression in an unpressurized device, $1 \mathrm{~h}$ of tissue compression resulted in significant damage but significantly less damage than the conventional method (Fig. 5d). We hypothesize that the increase in tissue damage in our device could be caused mainly by the sudden compression of the tissue during prolonged exposure to a static high-pressure valve. Although the cell death levels were normalized and are useful to compare across different platforms, one must be careful interpreting these data. Tissue thickness irregularities do not allow us to focus evenly across the tissue section and prevent us from obtaining quantitative values of cell death. Overall, our valve-mediated device showed less cell damage than a static conventional method.

\section{Drug dose-response assay}

Toxicological assays study the action mechanism and effects associated with the exposure of biological samples to chemical agents. Particularly, in ex vivo assays, a sample is exposed to increasing concentrations $(0.01 \mu \mathrm{M}-1 \mathrm{mM})$ of a chemical agent for short (min-h) or long (days) periods, depending on the drug action mechanism, cell signaling pathway or cellular metabo$\mathrm{lism}^{31-33}$. However, conventional toxicological assays require individual samples for each condition or concentration tested, limiting their applicability ${ }^{7,13}$. We tested the functionality of our device to deliver multiple concentrations of a drug to a single tissue sample. As a proof of concept, we used potassium cyanide (KCN), a drug that inhibits the production of adenosine triphosphate (ATP) in the mitochondrial respiratory chain, inducing cell apoptosis $^{34,35}$. The tissue sections were exposed to six different concentrations of $\mathrm{KCN}(0,0.1,1,5,10,50$, and $100 \mu \mathrm{M}$ ) or $0.05 \%$ Tween 20 as a positive control at flow rates of $2.5 \mu \mathrm{L} / \mathrm{min}$ for $30 \mathrm{~min}$ with the valve actuated at $\sim 16 \mathrm{kPa}$. To exclude contamination effects from contiguous channels, the concentrations were injected randomly in each of the 8 assay microchannels. Apoptotic and damaged cells were identified using EthD. As can be observed in Fig. 6, the fluorescence intensity was not homogeneous within the same assay microchannel, which can be attributed to some tissue regions being out of focus due to thickness irregularities. To compensate for these differences, the data were normalized to the channel with

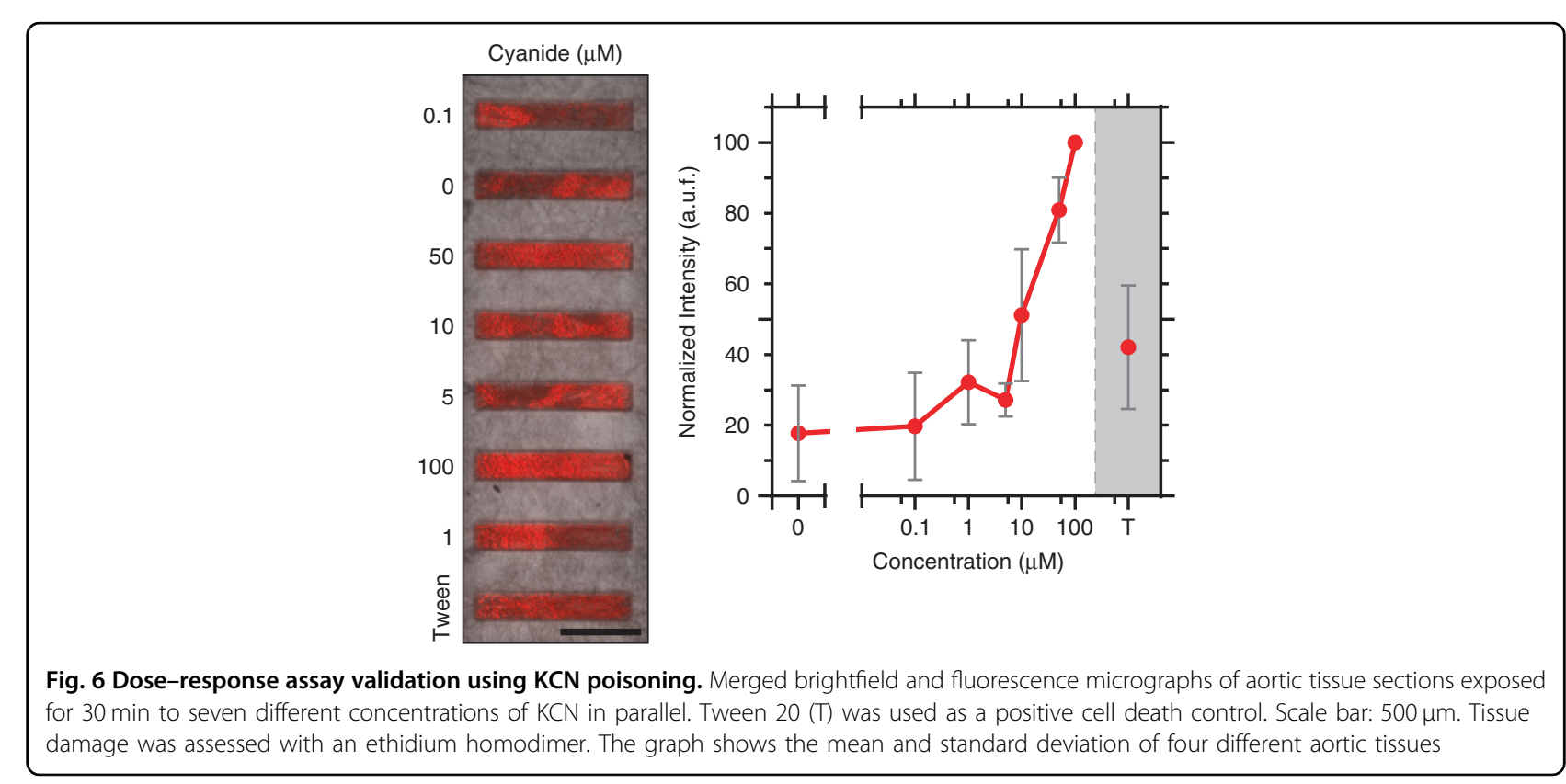


a

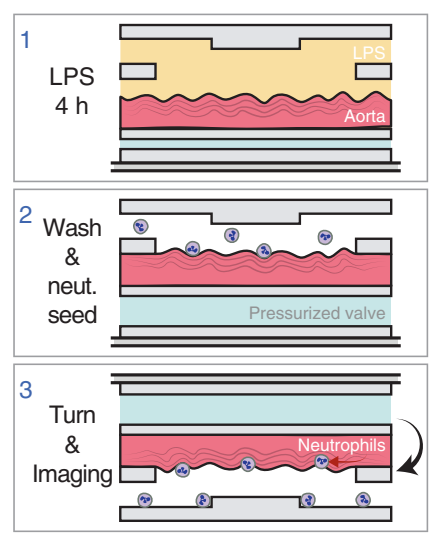

b

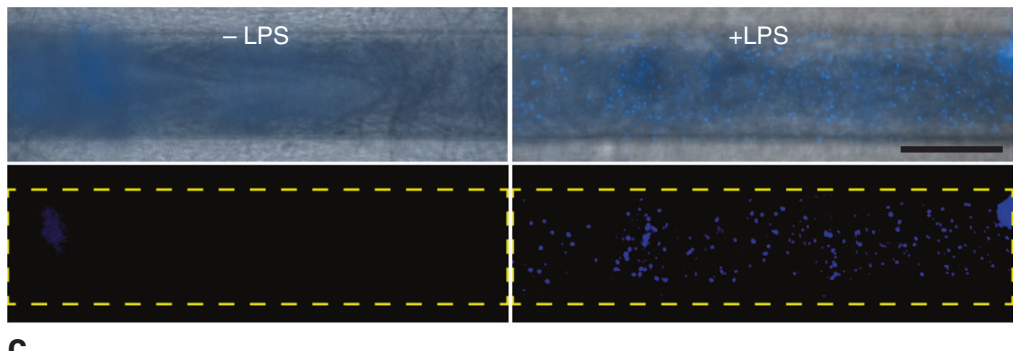

C
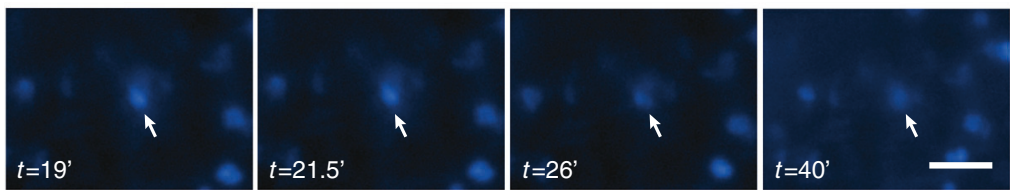

Fig. 7 Proinflammatory induction of an aorta. a Experimental setup. (1) The aorta was exposed to LPS for $4 \mathrm{~h}$. (2) After washing away LPS, the valve was actuated, and neutrophils (Hoechst stained) were perfused through the microchannels. (3) The device was flipped to allow unattached neutrophils to fall to the bottom surface and to later be removed. $\mathbf{b}$ Brightfield (top) and fluorescence (bottom) micrographs of the intima after exposure to buffer (left) or LPS (right). After $4 \mathrm{~h}$, under control conditions, no neutrophils adhered to the intima, while several neutrophils adhered to the endothelia after LPS exposure. Scale bar: $250 \mu \mathrm{m}$. c Top view micrographs of the intima. The arrows point to a single neutrophil extravasating the tunica intima at different time points $(\mathrm{min})$, revealed by its displacement along the Z-axis. Scale bar: $50 \mathrm{\mu m}$

the highest fluorescence signal, assuming that EthD penetrates evenly throughout the sample. As expected, the fluorescence intensity increased proportionally to $\mathrm{KCN}$ dose; however, for low $\mathrm{KCN}$ concentrations (0.1 and $1 \mu \mathrm{M})$, it was difficult to discriminate between tissue damage caused by manual manipulation $(0 \mu \mathrm{M})$ and drug effects (Figs. 6, S8). Although we expected similar levels of tissue damage between Tween 20 and the highest concentration of $\mathrm{KCN}$, the response difference between these two molecules can be attributed to the cellular mechanisms they attack. $\mathrm{KCN}$ is a small molecule $(65 \mathrm{Da})$ that diffuses rapidly into cells, blocking the mitochondrial respiratory chain, leading to an increase in radical oxygen species that damage the cell membrane much faster than Tween 20 and thus more effectively destroys cells ${ }^{35}$. In contrast, Tween $20(1.2 \mathrm{kDa})$ interacts poorly with membrane rafts called detergent-resistant membranes (DRMs), which slows cell membrane permeabilization ${ }^{36}$. In summary, this experiment indicated that different drug concentrations can be tested simultaneously in a single ex vivo tissue section, albeit for short periods of time (min-h).

\section{Proinflammatory response of the tunica intima}

Ex vivo studies open possibilities to mimic tissue inflammation and tumor metastatic events, allowing for the real-time observation of migratory cells (e.g., leukocytes, tumor cells, or pathogens) interacting with the tissue sample under different conditions ${ }^{37,38}$. As a complete organ-response experiment, we investigated the proinflammatory response of the endothelium when exposed to E. coli LPS (Fig. 7a). Briefly, the endothelium initiates a proinflammatory response when it detects LPS through the TLR4, CD14, and MD2 complex ${ }^{39}$. After 4h, Eselectin, ICAM, and VCAM are expressed on the membrane of endothelial cells, enabling the recruitment of neutrophils, which extravasate the endothelium to reach the area of inflammation.

Two murine aorta sections were incubated in our device with either cell culture media or LPS for $4 \mathrm{~h}$, while human neutrophils were isolated, purified, and stained with Hoechst. Next, neutrophils flowed through the channels of the device. After $3 \mathrm{~min}$, the device was turned over to observe whether neutrophils had adhered to the tunica intima. In the LPS-stimulated tissue, we counted 272 activated neutrophils (Fig. 7b), some of which extravasated into the endothelium, while other cells moved across the tissue surface for over $30 \mathrm{~min}$ (Fig. 7c and Movie S1). After $40 \mathrm{~min}, 265$ activated neutrophils were still adhered to the tissue surface of one of the microchannels (Fig. S9). Reproducibility was assessed on two more aortas from different mice on different days, identifying 48 and 13 neutrophils after $40 \mathrm{~min}$ of incubation (Figs. S10 and S11). Importantly, we did not observe the adhesion of neutrophils under control conditions (no LPS) in the three experiments performed. In summary, our microfluidic device can be used to study cell-tissue interactions and could be employed to investigate the intra- and extravasation mechanisms of inflammatory cells and circulating tumor cells.

\section{In vitro experimentation}

Fluorescence immunohistochemical staining in a fixed tissue

Fluorescence immunohistochemistry (IHC) staining has been a critical tool in biomedicine and in the clinic for the 
identification and diagnosis of diseases ${ }^{40}$. This technique identifies proteins or antigens by either using labeled antibodies with high specificity (direct method) or primary antibodies followed by labeled secondary antibodies (indirect method). A crucial step in IHC is the preservation of the tissue with a fixative agent such as formaldehyde. Formaldehyde preserves tissues but also enables detergent permeabilization without cellular degradation, allowing antibodies to reach their specific intracellular protein target. We adapted an IHC staining protocol in our microfluidic device to verify the device's ability to perform multiple staining assays in a single sample tissue.

Fresh tissue (an aorta) was placed in the device and subjected to the following steps: fixation, permeabilization, blocking, staining and washing. The valve was kept actuated at $\sim 34 \mathrm{kPa}$ during the experiment. There was neither leakage nor diffusion of dyes (verified with Dextran- $\mathrm{R}$ and Hoechst, respectively) outside the assay microchannels after $10 \mathrm{~min}$ of perfusion (Fig. 8a-1), even though the tissue was fixed and permeabilized on-chip. To confirm that multiple staining steps can indeed be performed in the same location, we washed all the microchannels with PBS, and the valve was deactivated for 2 min (Fig. 8a-2). Next, the valve was actuated, and Sytox Green was flowed through all the channels (Fig. 8a-3). Notably, the fluorescence signals from all the channels colocalized to the fluorescence signal measured in the previous step, confirming that the tissue remains in the same position and thus allowing for multiple staining steps in the same channel (Fig. 8b). Finally, the tissue section can be recovered for downstream high-resolution imaging or molecular analysis by easily peeling off the top and bottom PDMS layers (Fig. 8a-4).

Next, we conducted an indirect IHC staining assay for Von Willerbrand factor (VWF) on a fresh fixed aorta in our device. VWF is a protein that plays a role in blood coagulation and wound healing and can be used as a biomarker to identify functional endothelial cells ${ }^{41}$. We conducted four different IHC assays on a single aortic tissue: (i) a control with no antibodies, (ii) incubation with fluorescently labeled secondary antibodies, (iii) incubation with primary antibodies, and (iv) a combination of primary and secondary antibodies (Fig. 8c). In all cases, cell nuclei were stained with Sytox Green. As expected, after incubation for $1 \mathrm{~h}$, only the fourth condition showed a fluorescence signal in the red channel (corresponding to the fluorescent-labeled antibody), while the other conditions only presented a fluorescence signal in the green channel (Sytox Green) (Fig. 8c, d). No fluorescence signal was observed between channels. Furthermore, subcellular structures such as the nuclei (Fig. 8b, e) and VWF storage granules (Fig. 8e) can be clearly distinguished. Our approach reduces the number of tissue samples needed to carry out different assays and decreases IHC incubation times by at least fourfold compared with conventional protocols ${ }^{5,25,42}$. In summary, our device can perform indirect IHC staining assays; although only demonstrated for VWF, other proteins could be individually addressed in each assay channel, such as ICAM and ZO1.

\section{Conclusion}

Ex vivo conventional techniques rely on static environments that alter nutrient supply and create oxygen gradients, detrimental for tissue culturing and impeding parallel experimentation on a single tissue sample ${ }^{2,11-14}$. Microfluidic devices have enabled the implementation of multiple assays on tissue sections, typically using slices with smooth surfaces, that nevertheless do not prevent crosstalk between channels ${ }^{13,19}$. We were able to overcome this problem by incorporating a tunable valve underneath the tissue section that, when actuated, creates eight independent channels running in parallel through the surface of the tissue. This strategy allowed us to conduct parallel assays over a single tissue sample unrestrained from its surface topography, preventing reagent leakage and diffusion to contiguous channels while being periperfused.

As demonstrated here, ex vivo tissue sections can be cultured for short periods of time $(1 \mathrm{~h})$, enough time to perform relevant ex vivo and in vitro assays. Although in its current configuration, our device cannot be used for long-term culture periods $(>1 \text { day })^{15,23,43}$, this can be overcome by fitting a porous membrane in the tissue chamber to increase the delivery of nutrients to the adventitia (bottom side of the tissue). In addition, small microchannels could be patterned between each assay microchannel to increase the delivery of nutrients to the apical side of the tissue ${ }^{12,13}$. Small molecules might diffuse into adjacent microchannels during long incubation periods $(>1 \mathrm{~h})$, but this can be mitigated using the same strategy: patterning intercalating channels to limit diffusion into contiguous assay microchannels. Furthermore, these intercalated microchannels will decrease contact between the tissue surface and the PDMS, reducing cell compression and thus cell death.

For ex vivo experimentation, we carried out tissue viability assays that showed a lower cell death signal in the microfluidic device than on static conventional culture plates. As a proof of concept for toxicological assays, different concentrations of cyanide were delivered simultaneously to a tissue section without crosscontamination between the eight microchannels. Nevertheless, the device must be further validated by exposing a single tissue to different drugs in parallel. We also demonstrated proinflammatory experiments in which neutrophils interacted with a tissue section, which could be easily extended to other assays, such as pathogen-tissue interactions ${ }^{44}$. Furthermore, we also 


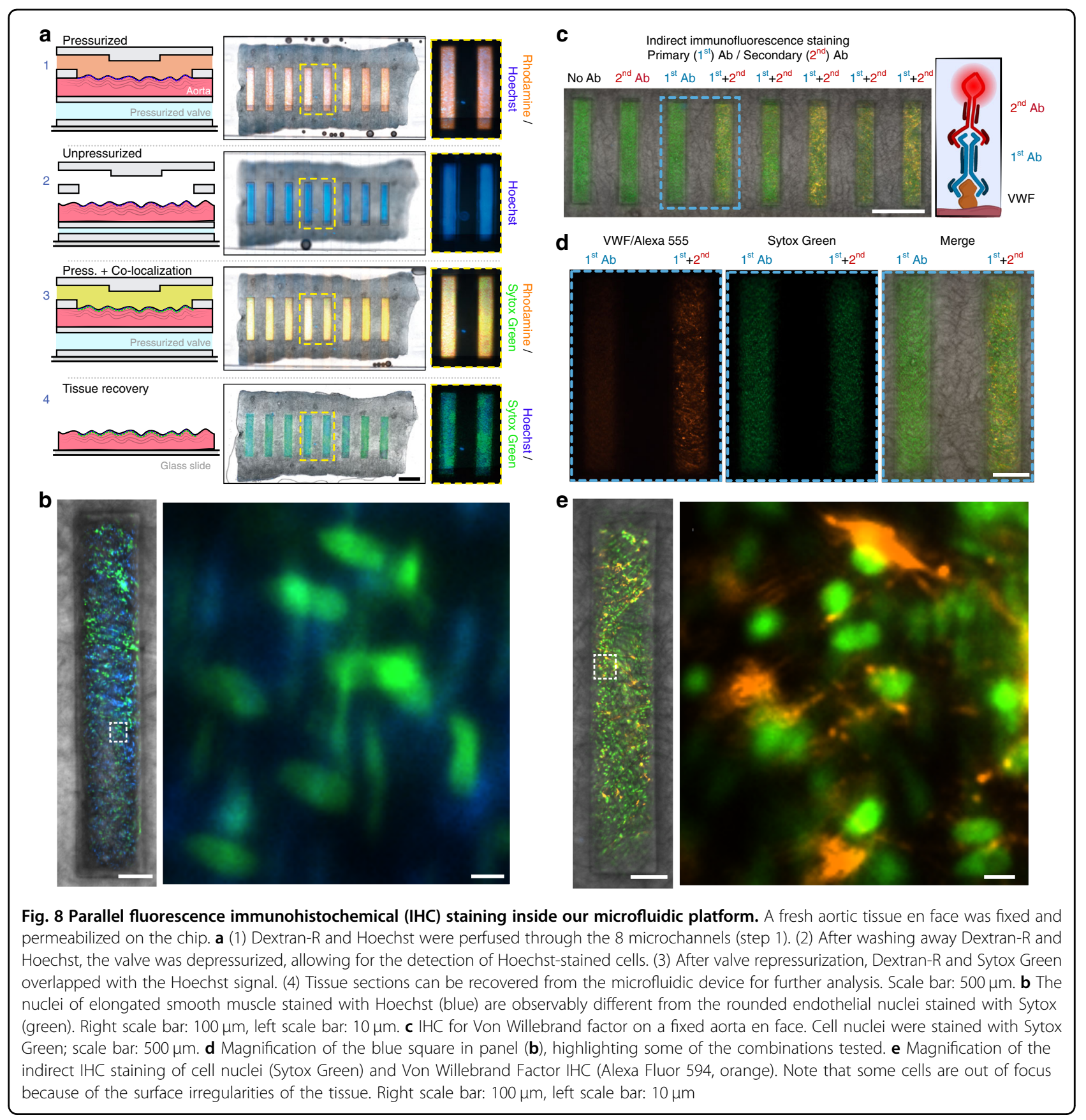

performed complete immunohistochemical staining assays, a typical in vitro assay. We believe our device can be used to solve other relevant biological questions, such as tissue remodeling and restoration after tissue injury or revascularization $^{45,46}$, and in biophysical tests, such as the study of mechanical compression effects (hypertensive in vitro model $)^{43}$.

There are a couple of strategies that can be implemented to image subcellular structures, currently hindered by the thickness of the PDMS layers. The first strategy is to decrease the thickness of the valve and flow layers to 1 and $3 \mathrm{~mm}$, respectively, which then offers the possibility of using long-distance objectives (6-12 $\mathrm{mm}$ working distance $)^{13}$. A second strategy is to disassemble the device, retrieve the tissue, and place it on a cover slip for downstream analysis using epifluorescence and confocal microscopes. Ultimately, the chosen strategy will depend on the type of assay implemented.

Tissue-cut slices are poised to become an important tool to test drug efficacy, safety, and toxicology in humans 
before clinical trials ${ }^{2,9,10}$. Moreover, they are expected to facilitate studies in the areas of physiology, electrophysiology, metabolism, tissue regeneration and scarring. Furthermore, ex vivo approaches such as organ slice cultures can bridge the gap between in vitro cell culture and in vivo mammalian models to advance our understanding of biology. This microfluidic device can also aid in diagnostics by reducing the volume of reagents needed to run different tests and by allowing multiple assays to be performed on a single sample. Developing tools to facilitate the interrogation of tissue sections, irrespective of their physical and mechanical characteristics, such as the one presented here, will be critical to further promote the widespread use of tissue slices.

\section{Materials and methods}

\section{Design and fabrication of the microfluidic device}

Master molds were fabricated using standard photolithography techniques. First, four silicon wafers (4", testgrade, Desert Silicon, USA) were cleaned in oxygen plasma (Zepto, Diener Electronic $\mathrm{GmbH}$ ). Next, the flow and valve master molds were created by spin-coating a 25$\mu \mathrm{m}$ layer of negative photoresist (GM1060, Gerstelec Sarl, Switzerland), while the tissue chamber and the assay microchannel master molds were coated with negative photoresist (SU-8 3050, MicroChem, USA) at the thicknesses of 250 and $150 \mu \mathrm{m}$, respectively. The wafers were prebaked as recommended by the manufacturer. The designs of each layer were patterned with a Micro Pattern Generator ( $\mu$ PG 101, Heidelberg Instruments). After a postbake step, the wafers were developed with PGMEA (484431, Sigma-Aldrich), followed by a hard-bake step of $2 \mathrm{~h}$ at $135^{\circ} \mathrm{C}$.

The microfluidic device was fabricated using multilayer soft lithography (Fig. S1). All master molds, acetate sheets (24075, Office Depot, Mexico) and steel press plates (diam.: $12 \mathrm{~cm}$, height: $2.5 \mathrm{~cm}$ ) were cleaned with isopropanol (V000139, Sigma-Aldrich) and blow-dried with $\mathrm{N}_{2}$ to eliminate any residues of PDMS or dust. The device is comprised of a top layer assembly (consisting of a flow layer and an assay microchannel layer) and a bottom layer assembly (composed of a tissue chamber layer and a valve layer). For the top assembly, a 10:1 weight ratio of PDMS elastomer and curing agent (Sylgard 184, Dow Corning) was mixed and poured on each of the molds. After degassing for $10 \mathrm{~min}$, excess PDMS was removed from the assay microchannel mold before being sandwiched with two acetate sheets and press plates to create a thick porous membrane. The flow layer was partially cured at $80^{\circ} \mathrm{C}$ for $21 \mathrm{~min}$ and cast out, and holes were punched and aligned to the assay microchannel layer. The assembly was cured for $2 \mathrm{~h}$ at $80^{\circ} \mathrm{C}$. For the bottom assembly, a weight ratio of 5:1 and 20:1 of elastomer to curing agent ratio was used for the valve and the tissue chamber layer, respectively. PDMS was poured on the tissue chamber mold and on the valve layer mold to heights of $500 \mu \mathrm{m}$ and $3 \mathrm{~mm}$, respectively, and cured at $80^{\circ} \mathrm{C}$ for $21 \mathrm{~min}$. After aligning the partially cured PDMS valve onto the tissue chamber layer, they were baked for $2 \mathrm{~h}$ at $80^{\circ} \mathrm{C}$. Next, inlets and outlets were punched out. During tissue dissection, the microchannel assay and tissue chamber layers were treated for $1.50 \mathrm{~min}$ with oxygen plasma before manually placing the tissue. The device is turned over and mounted on a motorized inverted microscope (Axio Observer A1, Zeiss Microscopy) to image the tissue surface.

\section{Imaging and statistical analysis}

Micrographs were analyzed using ImageJ (ver. 1.52, National Institutes of Health, USA) to obtain intensity values in all channels. GraphPad Prism (ver. 7; GraphPad Software) was used to statistically analyze the data obtained from micrographs.

\section{Tissue dissection and deposition}

All experiments with mice were approved by the Internal Committee of the Animal Care Facility of Cinvestav. The descending thoracic aortas and the trachea were dissected from healthy adult C57/B16 or C57BL/6$\mathrm{Tg}$ (CAG-EGFP)131Osb/LeySopJ mice after cervical dislocation. Connective tissue surrounding the organ was removed in cold DMEM/F12 (11330032, Gibco) with 2\% Pen/strep (15140-122, Thermo Fisher), followed by a transversal cut using microscissors (501233, McPhersonVannas Scissors, World Precision Instruments) to expose the inner surface wall en face.

The tips of flat forceps (503233, Dumont Forceps, World Precision Instruments) were submerged in a 10:1 PDMS base-to-curing agent ratio. The forceps were placed inside the oven for $1 \mathrm{~h}$ at $80^{\circ} \mathrm{C}$ in a vertical position with the tips pointing up and cured. This thin layer of PDMS prevents tissue adhesion and protects the tissue from forceps surface irregularities. Afterward, the forceps were submerged in alcohol and rinsed with 1X PBS before usage. The tissue was carefully placed on top of the flat PDMS-covered tip with the help of fine forceps (500342, Dumont \#5, World Precision Instruments). Tissues were held from their corners with fine forceps to minimize tissue damage. Next, most of the liquid from the tissue was carefully drained by capillarity through the tips of the fine forceps. Then, the tissue was manually placed in the tissue chamber before sealing the top and bottom layer of the device. Finally, media was flowed in the flow channel of the tissue chamber layer.

\section{Histology}

Aortas and tracheas en face were pinned to the surface of a petri dish coated with PDMS (10:1 ratio) using two 
acupuncture needles (PMX-GP, DBC, Korea) and were incubated overnight at $4{ }^{\circ} \mathrm{C}$ in $10 \%$ formalin (HT5011, Sigma) for fixation. Afterward, the aortas were frozen using PELCO Cryo-Embedding Compound (27300, Ted Pella), and 24- $\mu \mathrm{m}$-thick slices were cut using a cryostat (CM 1100-3, Leica). Phase-contrast and fluorescence images were acquired with a motorized inverted microscope (Axio Observer A1, Zeiss Microscopy).

\section{Precision-cut tissue slices}

After cervical dislocation, the murine brain was excised and cut into $2 \times 6 \mathrm{~mm}$ blocks in cold PBS. Then, the tissue blocks were embedded in agarose (A9539, Sigma-Aldrich) and solidified for $15 \mathrm{~min}$ at $4{ }^{\circ} \mathrm{C}$. Using a vibratome $(752-$ 888, Campden Instruments LTD, UK), 100- $\mu$ m-thick slices were cut from each tissue block in a cold bath of PBS. The tissue was gently collected and placed in the tissue chamber. The valve was pressurized at 3.4, 6.9, and $13.8 \mathrm{kPa}$, while dextran-rhodamine was flowed through the assay microchannels.

\section{Device characterization}

To characterize valve functionality, the assembly of the tissue chamber and the valve layers were bonded to a glass slide. A small PDMS slab $(5 \times 5 \mathrm{~mm})$ was plasma-bonded on top of the valve inlet, and a hole was punched, through which blue food dye was injected. The valve maximum operating pressure was characterized by increasing the pressure in $6.9 \mathrm{kPa}$ increments until the valve detached from the PDMS. Increasing membrane elevations were recorded with a USB digital microscope (Microcapture Pro 5MP, Celestron) from a side view of the valve. To study the elevation of the valve inside the tissue chamber, a finer characterization was conducted in steps of $0.7 \mathrm{kPa}$ using a thin PDMS cylinder placed on the center of the valve. Micrographs were taken after pressure actuation.

To characterize the crosstalk between microchannels and detect any leaks between microchannels, dextranconjugated rhodamine ( $40 \mathrm{kDa}, \mathrm{D} 1842$, Thermo Fisher) and fluorescein-5-isothiocyanate (389 Da, F7250, SigmaAldrich) were alternated in the 8 assay microchannels. For intercellular diffusion, fluorescent nuclear stains such as DAPI (62247, Thermo Fisher), Hoechst (H3570, Thermo Fisher) or Sytox Green (S7020, Thermo Fisher) were used in all assay microchannels. The valve was pressurized at $34.5 \mathrm{kPa}$.

\section{Tissue viability assay}

Two aortic tissue culturing methods were tested for tissue viability: (1) aorta en face, fixed with acupuncture needles on a Petri dish, and (2) aorta en face in our microfluidic device. Ethidium homodimer (EthD-1, L3224, Thermo Fisher) was used as an indicator of cell death. Tissue media consisted of DMEM/F12 with $2 \%$ pen/strep. After dissection in fresh tissue media, the tissue was directly exposed for 10 min to media with EthD-1 at $37^{\circ} \mathrm{C}$ and $5 \% \mathrm{CO}_{2}$ followed by micrograph acquisition. Next, the tissue was cultured in tissue media for $7 \mathrm{~h}$. Every hour, the tissue was exposed to EthD-1, and micrographs were acquired. As a positive cell death control, the tissue was exposed to DMEM with $0.05 \%$ Tween 20 (P9416, Sigma-Aldrich) for $10 \mathrm{~min}$.

To study whether valve actuation affected tissue viability, aortas were placed in a device, and the valve was pressurized at a physiological systolic pressure of a murine aorta of $16.2 \mathrm{kPa}^{47}$ for $1 \mathrm{~h}$ at $37^{\circ} \mathrm{C}$ and $5 \% \mathrm{CO}_{2}$. Next, the valve was deactivated, and tissue media with EthD-1 was perfused into the tissue chamber for $10 \mathrm{~min}$ before imaging.

After analyzing micrographs, the data were normalized by subtracting the difference in EthD- 1 intensity signals at $t=0^{\prime}$ (cell damage due to tissue dissection and manipulation) and after EthD-1 exposure $(t=\Delta)$ and by dividing the result by the highest intensity value per experiment.

\section{Drug dose-response assay}

A tunica intima sample was exposed to different concentrations of potassium cyanide (KCN) (60178, SigmaAldrich) with the valve actuated at $16.20 \mathrm{kPa}$. Each assay microchannel was perfused with $0,2,10,20,40,80$, or $100 \mathrm{mM} \mathrm{KCN}$ in tissue media for $30 \mathrm{~min}$ at $37^{\circ} \mathrm{C}$ and $5 \%$ $\mathrm{CO}_{2}$. The 8th assay microchannel was used as a positive control for cell death by flowing $0.05 \%$ Tween 20 . Finally, all assay microchannels were incubated for $10 \mathrm{~min}$ with EthD-1 and Hoechst.

To quantitate cell damage, we measured the average EthD-1 signal for each channel at two time points. First, immediately after placing the tissue in the device, $\mathrm{E}_{t=0}$, which is important to consider, as there can be significant tissue damage due to tissue dissection and manipulation. Then, after $30 \mathrm{~min}$ of incubation with the stimuli, $\mathrm{E}_{t=30}$. Next, we subtracted $\mathrm{E}_{t=30}$, from $\mathrm{E}_{t=0}$ for each channel and normalized this value to the channel with the highest intensity, which corresponded to the highest concentration of $\mathrm{KCN}$.

\section{Proinflammatory response of the aorta inside the microfluidic device}

A dissected descending aorta was cut in half, and each piece was placed in separate microfluidic devices. Inside the devices, one aorta en face was perfused for $4 \mathrm{~h}$ with tissue media containing $10 \mu \mathrm{g} / \mathrm{mL}$ lipopolysaccharide (LPS) from E. coli (L2755, Sigma-Aldrich, USA), while the second aorta was perfused with media as a control. The experiments were performed for $4 \mathrm{~h}$ at $37^{\circ} \mathrm{C}$ and $5 \% \mathrm{CO}_{2}$. Human neutrophils were isolated and purified from healthy donors as described elsewhere ${ }^{48}$. Purified neutrophils were stained with Hoechst and washed three 
times with Hank's balanced salt solution (HBSS, ICN1810454, MP Biomedicals). LPS was washed out from the tissue chamber using fresh tissue media. The valve was actuated at $16.2 \mathrm{kPa}$ before perfusing neutrophils into the assay microchannels at a flow rate of $2.5 \mu \mathrm{L} / \mathrm{min}$. After $5 \mathrm{~min}$, the flow was stopped, and the device was turned upside-down for video recording (Camtasia, TechSmith Corp.). Micrographs were captured every $15 \mathrm{~s}$. Neutrophil identification and quantification analysis was performed using ImageJ. Brightness and contrast from the fluorescence micrographs were adjusted until the Hoechst signal from label cells was visible and the background signal was removed. Each labeled cell was numbered and identified through the whole stack of images.

\section{Immunohistochemical staining}

Aortic tissue was fixed inside a microfluidic device with the valve actuated $(34.5 \mathrm{kPa})$ using $10 \%$ formalin for $24 \mathrm{~h}$ at $4{ }^{\circ} \mathrm{C}$. The valve remained actuated for the rest of the experiments. Then, the tissue was washed with tris (hydroxymethyl)aminomethane (TBS) buffer through the 8 assay microchannels, permeabilized with $0.05 \%$ Tween 20 for $30 \mathrm{~min}$, and blocked with casein blocking solution (37582, Thermo Fisher) supplemented with $2 \%$ bovine serum albumin (BSA, A9647, Sigma-Aldrich) for $10 \mathrm{~min}$. Next, the 8 microchannels were incubated for $3 \mathrm{~h}$ with the primary antibody against von Willerbrand factor (ab6994, Abcam). After washing, the assay microchannels were further incubated with Alexa-labeled secondary antibody (A-11012, Thermo Fisher) and Sytox Green for $1 \mathrm{~h}$. Staining controls for the primary and secondary antibodies were performed in the same tissue by omitting their respective antibodies.

\section{Acknowledgements \\ This work was funded by Mexico's National Council of Science and Technology (CONACyT) under Grants No. CB-256097, CB-286368, ERC-297690, and LN- 299051 and by Cinvestav Grant No. SEP FIDSC2018/104. We would like to thank Dr. Arturo Hernandez-Cruz (UNAM), Dr. Bruno Escalante, Dra. Ana Gámez-Méndez, Amelia Rios, and members of the Bio-ARTS laboratory for fruitful discussions. Supplementary information for the manuscript is available on the Microsystems \& Nanoengineering website http://www.nature.com/ micronano. \\ Author contributions \\ J.M.d.H.V. planned experiments, designed and fabricated the microfluidic device, performed experiments and data analysis, and wrote the paper. A.M.G. S. contributed with neutrophil experiments, data analysis and manuscript writing. J.L.G.C. designed experiments, supervised research and wrote the} paper. All authors read and approved the paper.

\section{Conflict of interest}

The authors declare that they have no conflict of interest.

Supplementary information accompanies this paper at https://doi.org/ 10.1038/s41378-020-0156-0.

Received: 9 August 2019 Revised: 4 March 2020 Accepted: 12 March 2020 Published online: 29 June 2020

\section{References}

1. Krumdieck, C. L., dos Santos, J. E. \& Ho, K. J. A new instrument for the rapid preparation of tissue slices. Anal. Biochem. 104, 118-123 (1980).

2. Gähwiler, B. H., Capogna, M., Debanne, D., McKinney, R. A. \& Thompson, S. M. Organotypic slice cultures: A technique has come of age. Trends Neurosci. 20, 471-477 (1997)

3. Graaf, I. A., de, Groothuis, G. M. \& Olinga, P. Precision-cut tissue slices as a tool to predict metabolism of novel drugs. Expert Opin. Drug Metab. Toxicol. 3, 879-898 (2007).

4. Ataç, B. et al. Skin and hair on-a-chip: in vitro skin models versus ex vivo tissue maintenance with dynamic perfusion. Lab Chip 13, 3555-3561 (2013).

5. Kim, M. S. et al. Breast cancer diagnosis using a microfluidic multiplexed immunohistochemistry platform. PLOS ONE 5, e10441 (2010).

6. Sivashankar, S. et al. Culturing of transgenic mice liver tissue slices in threedimensional microfluidic structures of PEG-DA (poly(ethylene glycol) diacrylate). Sens. Actuators, B Chem. 176, 1081-1089 (2013).

7. Astolfi, M. et al. Micro-dissected tumor tissues on chip: An ex vivo method for drug testing and personalized therapy. Lab Chip 16, 312-325 (2016).

8. Hill, M. R. H. \& Greenfield, S. A. The membrane chamber: a new type of in vitro recording chamber. J. Neurosci. Methods 195, 15-23 (2011).

9. Shamir, E. R. \& Ewald, A. J. Three-dimensional organotypic culture: experimental models of mammalian biology and disease. Nat. Rev. Mol. Cell Biol. 15, 647-664 (2014).

10. De Kanter, R. et al. Prediction of whole-body metabolic clearance of drugs through the combined use of slices from rat liver, lung, kidney, small intestine and colon. Xenobiotica 34, 229-241 (2004).

11. Schumacher, $\mathrm{K}$. et al. Perfusion culture improves the maintenance of cultured liver tissue slices. Tissue Eng. 13, 197-205 (2007).

12. Van Midwoud, P. M., Groothuis, G. M. M., Merema, M. T. \& Verpoorte, E. Microfluidic biochip for the perifusion of precision-cut rat liver slices for metabolism and toxicology studies. Biotechnol. Bioeng. 105, 184-194 (2010).

13. Chang, T. C. et al. Parallel microfluidic chemosensitivity testing on individual slice cultures. Lab Chip 14, 4540-4551 (2014).

14. Huang, Y., Williams, J. C. \& Johnson, S. M. Brain slice on a chip: opportunities and challenges of applying microfluidic technology to intact tissues. Lab Chip 12, 2103-2117 (2012)

15. Yamawaki, H., Lehoux, S. \& Berk, B. C. Chronic physiological shear stress inhibits tumor necrosis factor-induced proinflammatory responses in rabbit aorta perfused ex vivo. Circulation 108, 1619-1625 (2003).

16. Hematti, P. Mesenchymal stromal cells and fibroblasts: a case of mistaken identity? Cytotherapy 14, 516-521 (2012).

17. Maiellaro, K. \& Taylor, W. R. The role of the adventitia in vascular inflammation. Cardiovasc. Res. 75, 640-648 (2007).

18. Ross, A. E., Belanger, M. C., Woodroof, J. F. \& Pompano, R. R. Spatially resolved microfluidic stimulation of lymphoid tissue: ex vivo. Analyst 142, 649-659 (2017).

19. Shim, S., Belanger, M. C., Harris, A. R., Munson, J. M. \& Pompano, R. R. Two-way communication between ex vivo tissues on a microfluidic chip: application to tumor-lymph node interaction. Lab Chip 19, 1013-1026 (2019).

20. McLean, I. C., Schwerdtfeger, L. A., Tobet, S. A. \& Henry, C. S. Powering ex vivo tissue models in microfluidic systems. Lab Chip 18, 1399-1410 (2018).

21. Mohammed, J. S., Caicedo, H. H., Fall, C. P. \& Eddington, D. T. Microfluidic addon for standard electrophysiology chambers. Lab Chip 8, 1048-1055 (2008).

22. Dawson, A. et al. A microfluidic chip based model for the study of full thickness human intestinal tissue using dual flow. Biomicrofluidics 10, 064101 (2016).

23. Komeya, M. et al. Long-term ex vivo maintenance of testis tissues producing fertile sperm in a microfluidic device. Sci. Rep. 6, 1-10 (2016).

24. Kwon, S., Cho, C. H., Lee, E. S. \& Park, J. Automated Measurement of Multiple Cancer Biomarkers Using Quantum-Dot-Based Microfluidic Immunohistochemistry. Anal. Chem. 87, 4177-4183 (2015).

25. Ciftlik, A. T., Lehr, H.-A. \& Gijs, M. A. M. Microfluidic processor allows rapid HER2 immunohistochemistry of breast carcinomas and significantly reduces ambiguous (2+) read-outs. Proc. Natl Acad. Sci. USA 110, 5363-5368 (2013).

26. Bellini, $C$. et al. Comparison of 10 murine models reveals a distinct biomechanical phenotype in thoracic aortic aneurysms. J. R. Soc. Interface 14, 20161036 (2017).

27. Jeong, J. Y. et al. Functional and developmental analysis of the blood-brain barrier in zebrafish. Brain Res. Bull. 75, 619-628 (2008).

28. Ambati, J. et al. Diffusion of high molecular weight compounds through sclera. Investig. Ophthalmol. Vis. Sci. 41, 1181-1185 (2000). 
29. Leddy, H. A. \& Guilak, F. Site-specific effects of compression on macromolecular diffusion in articular cartilage. Biophys. J. 95, 4890-4895 (2008).

30. Shi, Z.-D. \& Tarbell, J. M. Fluid flow mechanotransduction in vascular smooth muscle cells and fibroblasts. Ann. Biomed. Eng. 39, 1608-1619 (2011).

31. Wu, M. L. et al. Short-term resveratrol exposure causes in vitro and in vivo growth inhibition and apoptosis of bladder cancer cells. PLOS ONE 9 (2014).

32. Gerlach, M. M. et al. Slice cultures from head and neck squamous cell carcinoma: A novel test system for drug susceptibility and mechanisms of resistance. Br. J. Cancer 110, 479-488 (2014).

33. Behrsing, H. P., Furniss, M. J., Davis, M., Tomaszewski, J. E. \& Parchment, R. E. In vitro exposure of precision-cut lung slices to 2-(4-amino-3-methylphenyl)-5fluorobenzothiazole lysylamide dihydrochloride (NSC 710305, Phortress) increases inflammatory cytokine content and tissue damage. Toxicol. Sci. 131, 470-479 (2013).

34. Zhang, Y. et al. AMP-activated protein kinase is involved in endothelial NO synthase activation in response to shear stress. Arterioscler. Thromb. Vasc. Biol. 26, 1281-1287 (2006)

35. Prabhakaran, K., Li, L., Borowitz, J. L. \& Isom, G. E. Caspase inhibition switches the mode of cell death induced by cyanide by enhancing reactive oxygen species generation and PARP-1 activation. Toxicol. Appl. Pharmacol. 195 194-202 (2004)

36. Dimitrijevic, D., Shaw, A. J. \& Florence, A. T. Effects of Some Non-ionic Surfactants on Transepithelial Permeability in Caco-2 Cells. J. Pharm. Pharmacol. 52, 157-162 (2000).

37. Marie-th, $\mathrm{S}$. et al. Age-specific function of a5 $\beta 1$ integrin in microglial migration during early colonization of the developing mouse cortex. Glia $\mathbf{6 5}, 1072-1088$ (2017).
38. Stoletov, K. et al. Visualizing extravasation dynamics of metastatic tumor cells. J. Cell Sci. 123, 2332-2341 (2010).

39. Dauphinee, S. M. \& Karsan, A. Lipopolysaccharide signaling in endothelial cells. Lab. Investig. 86, 9-22 (2006).

40. Cors, J. F., Kashyap, A., Khartchenko, A. F., Schraml, P. \& Kaigala, G. V. Tissue lithography: Microscale dewaxing to enable retrospective studies on formalinfixed paraffin-embedded (FFPE) tissue sections. PLOS ONE 12, 1-14 (2017).

41. Sadler, J. E. et al. Update on the pathophysiology and classification of von Willebrand disease: a report of the Subcommittee on von Willebrand factor. J. Thromb. Haemost. 4, 2103-2114 (2006).

42. Kim, M. S., Kwon, S., Kim, T., Lee, E. S. \& Park, J. K. Quantitative proteomic profiling of breast cancers using a multiplexed microfluidic platform for immunohistochemistry and immunocytochemistry. Biomaterials 32, 1396-1403 (2011).

43. Prasad, R. M., Jin, X., Aboualaiwi, W. A. \& Nauli, S. M. Real-time vascular mechanosensation through ex vivo artery perfusion. Biol. Proced. Online 16, 1-7 (2014).

44. Yissachar, N. et al. An intestinal organ culture system uncovers a role for the nervous system in microbe-immune crosstalk. Cell 168, 1135-1148.e12 (2017).

45. Ghoochani, A. et al. A versatile ex vivo technique for assaying tumor angiogenesis and microglia in the brain. Oncotarget 7, 1838-1853 (2016).

46. Poosti, F. et al. Precision-cut kidney slices (PCKS) to study development of renal fibrosis and efficacy of drug targeting ex vivo. Dis. Model. Mech. 8, 1227-1236 (2015).

47. Mattson, D. L. Comparison of arterial blood pressure in different strains of mice. Am. J. Hypertens. 14, 405-408 (2001).

48. Jimenez-Valdes, R. J., Rodriguez-Moncayo, R., Cedillo-Alcantar, D. F. \& GarciaCordero, J. L. Massive parallel analysis of single cells in an integrated microfluidic platform. Anal. Chem. 89, 5210-5220 (2017). 